\title{
Rothmund-Thomson Syndrome
}

National Cancer Institute

\section{Source}

National Cancer Institute. Rothmund-Thomson Syndrome. NCI Thesaurus. Code C3335.

An autosomal recessive inherited syndrome usually caused by mutations in the RECQL4 gene. It is characterized by poikilodermatous skin changes, sparse hair, cataracts, small stature, skeletal abnormalities, and an increased predisposition to cancer, particularly osteosarcoma. 